\title{
Myalgia as the revealing symptom of multicore disease and fibre type disproportion myopathy
}

\author{
C Sobreira*, W Marques Jr, A A Barreira
}

J Neurol Neurosurg Psychiatry 2003;74:1317-1319

Background: Multicore disease and congenital fibre type disproportion myopathy are diseases assigned to the heterogeneous group of congenital myopathies. Although hypotonia and muscle weakness appearing in early life are the commonest manifestations of these diseases, distinct phenotypes and late onset cases have been described.

Objective: To report the occurrence of myalgia as the revealing symptom of multicore disease and fibre type disproportion myopathy.

Methods: The clinical cases of three patients with fibre type disproportion myopathy and one with multicore disease are described. Skeletal muscle biopsies were processed for routine histological and histochemical studies.

Results: The clinical picture was unusual in that the symptoms were of late onset and the predominant complaint was muscle pain exacerbated by exercise. Muscle weakness was found in only a single patient, the mother of a patient with fibre type disproportion myopathy. Physical examination was unremarkable in the other patients. Muscle biopsies from patients 1 and 2 contained type I fibres that were considerably smaller than the type II fibres, supporting the diagnosis of fibre type disproportion myopathy. Skeletal muscle of patient 4 showed multiple areas, predominantly but not exclusively in the type I fibres, from which oxidative enzyme activities were absent, as seen in multicore disease.

Conclusions: Muscle pain was the main clinical manifestation in our patients. Recognition of the broader clinical expression of these myopathies is important for prognostic reasons and for genetic counselling of the family members.

M ulticore disease and congenital fibre type disproportion myopathy (CFTDM) are diseases assigned to the group of congenital myopathies. They most commonly manifest during the neonatal period, usually as nonprogressive weakness and hypotonia. ${ }^{1}$ The main histopathological finding on muscle biopsy from patients with CFTDM is an isolated and uniform disproportion of fibre size, in which type I fibres are more than $15 \%$ smaller than type II fibres. Type I fibre predominance was also reported. Multiple areas of focal myofibrilar degeneration in both type I and type II fibres, extending two or three sarcomeres in length, which are devoid of mitochondria, constitute the distinguishing morphological finding observed in the skeletal muscles from patients with multicore disease. ${ }^{2}$

Unusual clinical manifestations have been reported in association with the morphological features that characterise certain congenital myopathies. Multicore disease may begin in adult life $e^{3-5}$ and distinct clinical features such as cardiomyopathy, ${ }^{6}$ ophthalmoplegia, ${ }^{78}$ arthrogryposis, ${ }^{9}$ and respiratory muscle involvement may be present. The symp- toms of CFTDM are more uniform. However, some patients exhibit unusual phenotypes such as rigid spine syndrome, ${ }^{10} 11$ significant dysmorphic features, ${ }^{12}$ or very mild symptoms. ${ }^{13}$

Cramps are uncommon complaints in patients with multicore disease or CFTDM and exercise related muscle pain has not been associated with multicore disease. Aimed at contributing to better delineating the phenotypic expression of these myopathies, we present the clinical cases of patients suffering late onset, generalised muscle pain, whose muscle biopsies revealed the distinguishing features of either multicore disease or CFTDM.

\section{CASE REPORTS \\ Patient 1}

A 24 year old man was referred to a neurologist owing to exercise related myalgia involving both the upper and lower limbs. Birth and early development were normal. The symptom began in childhood when a mild precocious fatigue limited his physical activities. In adolescence, muscle pain was felt shortly after physical efforts such as riding a bicycle. The symptoms progressed slowly. Currently, he suffers mild myalgia, even when at rest. Family history was negative for neurological diseases. Physical and neurological examinations were unremarkable, except that the patient was thin. Muscle weakness and hypotonia were absent. The electromyography (EMG) was normal. Serum creatine kinase (CK) was moderately increased (four times the upper normal limit) and myoglobinuria was not detected. Other laboratory tests performed to investigate metabolic dysfunction were normal. The pathological findings on sections of a frozen biceps brachii muscle biopsy were uniformly atrophic type I fibres, approximately $26 \%$ smaller than type II fibres, in the absence of other abnormal features (fig 1). A similar proportion of type I and type II fibres were present. A fibre type disproportion myopathy was diagnosed.

\section{Patients 2 and 3}

This 14 year old male, born of non-consanguineous parents, complained of exertional myalgia since 8 years of age with no apparent progression. The boy is unable to practice sports because of muscle pain and precocious fatigue. He also suffers muscle cramps after exercise. Early development was normal. His mother (patient 3) has suffered mild muscle pain on exertion since childhood. Clinical examination showed mother and son to be short in stature. The mother was thin, but the son was well built. Neurological examination failed to demonstrate muscle weakness in the son in whom only mild hypotonia was found. In the mother, mild weakness was detected in the neck flexors and proximal muscles of the lower limbs. The EMGs of mother and son showed myopathic

Abbreviations: CFTDM, congenital fibre type disproportion myopathy; CK, creatine kinase; EMG, electromyograph 

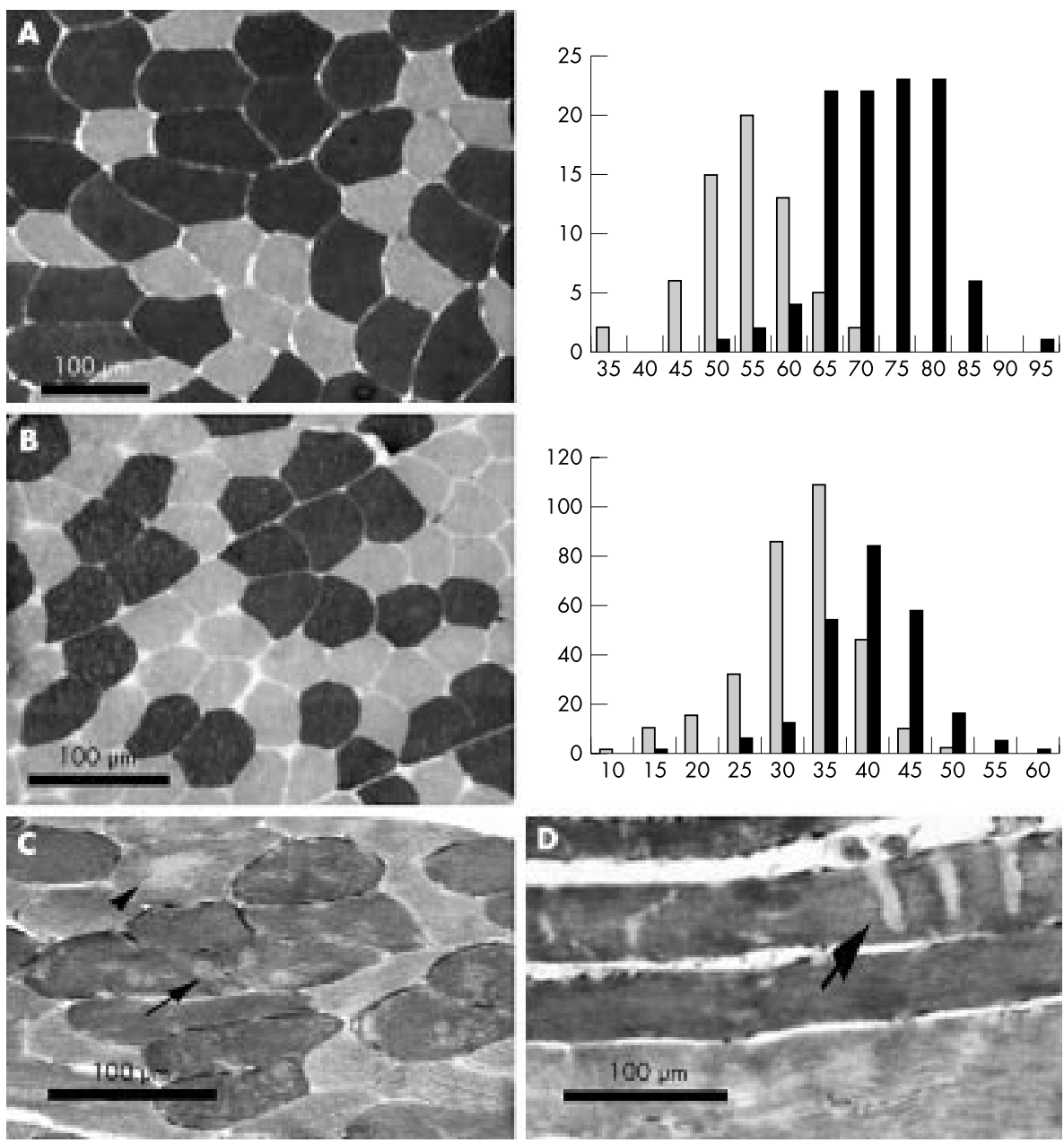

Figure 1 Frozen sections of left biceps brachii muscle biopsies from patients 1 (A) and 2 (B) revealing myofibrillar ATPase after preincubation at $\mathrm{pH}$ 9.4. Note the small, uniform type I fibres (light fibres). The corresponding histograms show on abscissa diameters in um and on ordinate number of fibres. Type I fibres are represented as light bars and type II fibres as dark bars. Quantitative analysis performed using the image analysis software Image-Pro Plus, version 4.1 for windows, Media Cybernetics, L.P. Transverse sections of a left biceps brachii biopsy from patient 4 (C) showing NADH-TR. Multiple areas from which enzyme activity is absent (multicores) are seen in type I (dark fibres: arrows) and type II fibres (arrowhead). Longitudinal sections (D) show that cores are limited to a few $\mu \mathrm{m}$ in length (arrows).

changes in the proximal arm and leg muscles. Serum CK was normal in both. Other ancillary tests performed to evaluate metabolic dysfunction were normal. Transverse cryostat sections of a biceps brachii biopsy showed the boy's type I fibres to be $21 \%$ smaller than his type II fibres (fig 1). Type I fibre predominance was also detected $(61 \%$ of fibres were type I). In the absence of additional abnormalities, the diagnosis of fibre type disproportion myopathy was established. Muscle biopsy from the mother was normal.

\section{Patient 4}

At age 40, a 47 year old woman complained of muscle pain at rest, initially in the neck extensors, followed by the scapular and pelvic girdles, and subsequently by the proximal limb regions, where pain is currently more intense. The pain was aggravated by mild physical efforts and precocious fatigue was reported. Early development was unremarkable. Family history disclosed a third degree relative with congenital presentation of multicore disease. When examined, seven years after onset of the disease, the patient was thin, with a hypotrophic musculature. Despite mild and generalised hypotonia, muscle strength was normal, tendon reflexes were preserved, and EMG was myopathic. Serum CK was normal as were other laboratory parameters such as C-reactive protein, mucoproteins, alpha 1 acid glycoprotein, serum electrolytes, thyroid function tests, and blood lactate. A biceps brachii biopsy revealed many fibres reduced in diameter and most type I and occasional type II fibres showing multiple, small, circular regions from which oxidative enzyme activity was absent (fig 1), findings that support the diagnosis of multicore disease. In some fibres a single and central area of absent oxidative enzyme activity resembled the central core lesion. There was also type I fibre predominance.

\section{DISCUSSION}

In this paper we describe patients with myalgia, whose muscle biopsies showed the pathological changes observed in either CFTDM or multicore disease. These cases are peculiar in that the occurrence of late onset muscle pain as either the only or predominant and most disabling symptom has been rarely reported in association with the above cited diseases. Muscle biopsy and a range of laboratory tests performed on our patients did not show any signs of acquired conditions associated with myalgia, such as inflammatory and metabolic myopathies, or endocrine diseases. EMG also confirmed the myopathic nature of their diseases.

Our patients' clinical phenotypes were not of a congenital myopathy, since manifestations began in late childhood or in adult life. Therefore it seems most appropriate to use the term fibre type disproportion myopathy to refer to the diseases of patients 1, 2, and 3. However, the morphological alterations in their skeletal muscles, together with the existence of affected 
family members indicating the probable genetic nature of the diseases, suggest a relationship with the classical, congenital myopathies manifesting similar pathological findings in muscle biopsy. Several reports of CFTDM have disclosed an autosomal dominant inheritance. ${ }^{14}{ }^{15}$ The family history of patients 2 and 3 also suggests an autosomal dominant trait, despite the normal appearance of muscle biopsy from patient 3. Since mother and son presented very similar clinical phenotypes, we could speculate that the discrepancies in the pathological findings could result from uneven involvement of different portions of the skeletal musculature in this disease, as previously demonstrated. ${ }^{14}$ Since many patients with CFTDM improve as they grow older, the disappearance of the characteristic pathological alteration in adulthood could also explain our findings. A previously well documented case of CFTDM with muscle pain as the predominant feature was described by Santoro et al. ${ }^{13}$ Their patient, a 9 year old girl, suffered from myalgia since she was seven. Muscle weakness was very slight. Congenital hypotonia, delayed acquisition of motor milestones, and skeletal deformities were absent. It is widely accepted that fibre type disproportion may be a feature of distinct genetic disorders. Therefore, it is probable that we are facing different disease entities. Determining the genetic defect in these patients will help to clarify the pathophysiological alterations that lead to fibre type disproportion.

Myalgia was not a feature in previously described patients with multicore disease. However, patients with muscle cramps have been identified. ${ }^{3}$ Our patient's complaints did not include cramps, consisting mainly of myalgia at rest, exacerbated by physical effort. The existence of an affected third degree relative with the classical phenotype of this congenital myopathy corroborates the diagnosis of our patient. Family tree analysis suggests an autosomal recessive trait. Although different patterns of inheritance have been reported in such disease, autosomal recessive inheritance predominates. ${ }^{16} 17$ The variable expression of multicore disease observed in some families ${ }^{18} 19$ may in part justify the various inheritance patterns associated with this congenital myopathy. Inheritance may be misinterpreted in families in which clinical examination and laboratory studies are not performed on all family members. Genetic heterogeneity, demonstrated in recent molecular genetic studies that reported mutations in two different genes in patients with multiminicore disease, the selenoprotein $\mathrm{N}$ gene, ${ }^{20}$ the same gene implicated in rigid spine muscular dystrophy, and the ryanodine recptor type 1 gene, ${ }^{21}$ may as well be responsible for the observed variability. The coexistence of classical and unusual cases within the same kindred strongly suggests that a single genetic defect may manifest as distinct phenotypes. The occurrence of symptomatic carriers may in part explain this phenomenon. The elucidation of the genetic defect in each family with multicore disease will help to identify the factors responsible for the existence of distinct phenotypes.

The signs and symptoms of the patients described here corroborate the existence of a wide range of clinical manifestations of multicore disease and fibre type disproportion myopathy. Since muscle pain at rest and exertional myalgia are non-specific features common to a variety of disorders, many cases of such diseases may remain unrecognised. Although the diagnosis of either affection may not necessarily lead to a specific treatment, the prevention of life threatening complications, the development of supportive and symptomatic therapies, and adequate information concerning prognosis can be provided to the patients. Genetic counselling is of major importance and should be offered to the families since more severe phenotypes may coexist in the same kindred.
Further elucidation of the nature of the morphological and genetic abnormalities associated with these diseases is crucial to defining whether the different manifestations reported to date represent clinical variations of the same disease, or result from similar responses of muscle to various adverse situations.

\section{ACKNOWLEDGEMENTS}

We are grateful to Dr JAM Oliviera and Dr LN Serafina for providing the stained tissue sections of the patients. Dr C Sobreira received financial support from the grant 99/ of FAPESP.

\section{Authors' affiliations}

C Sobreira, W Marques Jr, A A Barreira, Departamento de

Neurologia, Psiquiatria e Psicologia Médica, Universidade de São Paulo, Brazil

Correspondence to Dr Sobreira, Departamento de Neurologia Psiquiatria e Psicologia Médica, HC sala 536, Faculdade de Medicina de Ribeirão Preto, USP, Campus Universitário Monte Alegre, Ribeirão Preto-SP, Brazil, 14048-900; csobreira@rnp.fmrp.usp.br

Received 10 November 2002

In revised form 8 March 2003

Accepted 21 March 2003

\section{REFERENCES}

1 Fardeau M, Tome FMS. Congenital myopathies. In: Engel AG, Franzini-Armstrong C, eds. Myology. Basic and Clinical. New York: McGraw-Hill, Inc., 1994: 1487-1532

2 Bodensteiner, JB. Congenital Myopathies. Muscle Nerve 1994; 17:131-44

3 Bonnette H, Roelofs R, Olson WH. Multicore disease: report of a case with onset in middle age. Neurology 1974;24:1039-44.

4 Shuaib A, Martin JME, Mitchell B, et al. Multicore myopathy: not always a benign entity. Can J Neurol Sci 1988;15:10-14.

5 Zeman AZJ, Dick DJ, Anderson JR, et al. Multicore myopathy presenting in adulthood with respiratory failure. Muscle Nerve 1997;20:367-9.

6 Magliocco AM, Mitchell LB, Brownell AK, et al. Dilated cardiomyopathy in multicore myopathy. Am J Cardiol 1989;63:150-1.

7 Swash M, Schwartz MS. Familial multicore disease with focal loss of cross-striations and ophthalmoplegia. J Neurol Sci 1981:52:1-10.

8 Jungbluth $\mathbf{H}$, Sewry C, Brown SC, et al. Minicore myopathy in children: a clinical and histopathological study of 19 cases. Neuromuscul Disord 2000; 10:264-73

9 Ferreiro A, Estournet B, Chateau D, et al. Multi-minicore disease-searching for boundaries: phenotype analysis of 38 cases. Ann Neurol 2000;48:745-57.

10 Goebel HH, Lenard HG, Gorke W, et al. Fibre type disproportion in the rigid spine syndrome. Neuropediatrics 1977;8:467-77.

11 Seay AR, Ziter FA, Petajan JH. Rigid spine syndrome. A type I fiber myopathy. Arch Neurol 1977;34:1 19-22.

12 Sulaiman AR, Swick HM, Kinder DS. Congenital fibre type disproportion with unusual clinico-pathologic manifestations. J Neurol Neurosurg Psychiatry 1983;46:175-82.

13 Santoro L, Giudice E, Francica D, et al. Congenital fiber type disproportion myopathy. Report of a case with late onset and myalgia. Clin Pediatr 1985;24:219-20.

14 Cavanagh NPC, Lake BD, McMeniman P. Congenital fibre type disproportion myopathy. A histological diagnosis with an uncertain clinical outlook. Arch Dis Child 1979;54:735-43.

15 Eisler T, Wilson JH. Muscle fiber-type disproportion: report of a family with symptomatic and asymptomatic members. Neurology 1978;35:823-6

16 Gardner-Medwin D. Neuromuscular disorders in infancy and childhood. In: Walton JN, Karpati G, Hilton-Jones D, eds. Disorders of Voluntary Muscle. Edinburgh: Churchill-Livingstone, 1994:783-4.

17 Taratuto AL. Congenital myopathies and related disorders. Curr Opin Neurol 2002; 15:553-61

18 Vanneste JA, Stam FC. Autosomal dominant multicore disease. J Neurol Neurosurg Psychiatry 1982:45:360-5.

19 Joy JL, Oh SJ. Asymptomatic hyper-CK-emia: an electrophysiologic and histopathologic study. Muscle Nerve 1989;12:206-9.

20 Ferreiro A, Quijano-Roy S, Pichereau C, et al. Mutations of the selenoprotein $\mathrm{N}$ gene, which is implicated in rigid spine muscular dystrophy, cause the classical phenotype of multiminicore disease: reassessing the nosology of early-onset myopathies. Am J Hum Genet 2002;71:739-49.

21 Galli L, Orrico A, Cozzolino S, et al. Mutations in the RYR1 gene in Italian patients at risk for malignant hyperthermia: evidence for a cluster of novel mutations in the C-terminal region. Cell Calcium 2002;32:143. 